\title{
Driver Drowsiness Detection System for Vehicle Safety
}

\author{
A. Subbarao, K.Sahithya
}

\begin{abstract}
Detection of drowsiness of driver is a vehicle safety technology, which helps to put off accidents which caused by the driver being dozy. A variety of studies have recommended that around $20 \%$ of all road accidents are due to drowsiness of the driver. The developments of technologies for detecting or preventing drowsiness while driving is a major confront in accident evasion systems. Because of the peril of the tiredness while driving, different new methods need to be developed for counteracting the effect. The paper is based on a example for detection of drowsiness system. The intend of this paper is design of an automated system for safety of driver from improper driving. The system is designed such that it will precisely scrutinize the eye blink. In this paper, the eye blink of the driver is detected by using eye blink sensor which is IR based. The disparity across the eye will vary as per eye blink.The output is high, if the eye is closed or else output is low. It indicates closing or opening position of an eye. TheIR outputis given to circuit to signify the alarm. The controller will send a warning signal so that it is displayed on liquid crystal display screen. The buzzer, which is placed near the driver, will be activated and alters the driver when he falls asleep during driving. The alcohol sensor is also used to detect whether the driver is drunken which avoids accident caused by the drunken drivers. According to the intensity of light, the lights will be ON or OFF inside the vehicle, this saves power consumption. Tilt sensor is also used to detect whether the vehicle met with an accident or not.
\end{abstract}

KEYWORDS - LPC2148, Drowsiness detection, Eye blink sensor, GSM, GPS.

\section{INTRODUCTION}

Driver drowsiness driving is one of the main reasons for road accidents. In current survey it shows that out of 5 accidents one accident is due to drowsiness of the driver which is approximately $20 \%$ of road accidents and it increasing gradually in every year. The survey highlights the facts that total number of traffic deaths are excessive because of drowsiness of the driver. Driving a vehicle in a crowded road has become a nightmare because of the road conditions, poor weather conditions, haste to reach the destination and excess of traffic. Drowsiness of driver, drunk and drive are coming further major reasons for road accidents.Due to less conscious we can't take care of ours while driving. To provide security to driver, the vehicles are assisted with automated safety system that alerts driver by using alarm. All vehicles should be equipped with eye blink sensor and alcohol sensor sequentially to evade these types of accidents. The objective of the project is to by using IR sensor the eye blink is measured and controlled. The infrared rays are transmitted by IR transmitter into driver's eye. The eye reflects the transmitted infrared rays and these

Revised Manuscript Received on April 12, 2019.

Dr. A. Subbarao,Associate professor, ECE Department, SR Engineering College, Warangal, Telangana, India (E-mail: Email:subbarao_ka@yahoo.com.

K.Sahithya, M. Tech student, ECE Department, S.R Engineering College, Warangal, Telangana, India reflected rays are received by the IR receiver. If the eye is in closed status, the output of IR receiver is high. The IR receiver output is low, if the eye is in opening position. This informs that the eye is in opening or closing position. The alarm is indicated, if the output is given to logic circuit. This project is to decrease the accidents due to comatose through eye blink.

\section{EXISTING SYSTEM}

By using a non intrusive machine vision based concepts, drowsiness of the driver detected system is developed. Many existing systems require a camera which is installed in front of driver [4]. It points straight towards the face of the driver and monitors the driver's eyes in order to identify the drowsiness. For large vehicle such as heavy trucks and buses this arrangement is not pertinent. Bus has a large front glass window to have a broad view for safe driving. If we place a camera on the window of front glass, the camera blocks the frontal view of driver so it is not practical. If the camera is placed on the frame which is just about the window, then the camera is unable to detain the anterior view of the face of the driver correctly. The open $\mathrm{CV}$ detector detects only $40 \%$ of face of driver in normal driving position in video recording of 10 minutes. In the oblique view, the Open $\mathrm{CV}$ eye detector (CV-ED) frequently fails to trace the pair of eyes. If the eyes are closed for five successive frames the system concludes that the driver is declining slumbering and issues a warning signal [4]. Hence existing system is not applicable for large vehicles. In order to conquer the problem of existing system, new detection system is developed in this project work.

\section{PROPOSED SYSTEM}

In order to overcome this eye blink sensor is used. A spectacle with eye blink sensor is used to detect the driver drowsiness and alerts the driver with buzzer, if driver is affected by drowsiness. The various hardware components of project are mentioned below

1. LPC2148 microcontroller

2. Eyeblink sensor

3. Alcohol sensor

4. Tilt sensor

5. LDR

6. LCD and buzzer

7. GSM and GPS

The block diagram for the implemented project is shown in fig (1). 


\section{A. Power supply}

In this system, the power supply of $12 \mathrm{~V}$ is used for transmitter section and receiver section. For conversion of the A.C. to D.C rectifiers are being used. To step down the voltage, a step down transformer is used.

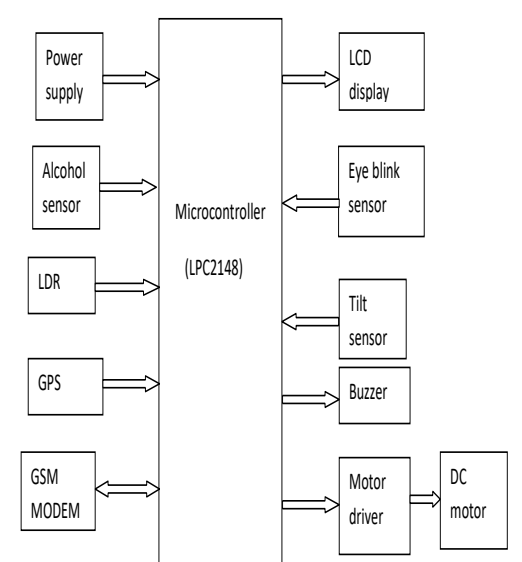

Fig1: Block diagram

\section{B. Microcontroller Section}

Here microcontroller section is the control unit of the project. It consists of a Microcontroller with its related circuitry like Crystal with capacitors, Pull up resistors, and reset circuitry and so on. The heart of the project is the microcontroller because it controls the interfaced devices. By using written program it communicates with the devices.

Here 16-bit/32-bit ARM7TDMI-S controller with a small leadless quad flat package. It supports up to 40KB RAM and $512 \mathrm{~KB}$ on chip flash ROM and supports crystal frequency of $60 \mathrm{MHz}$ used high speed operation.

\section{Eye blink sensor}

An eye blinking is mandatory in this work, since it is used to impel the device and to activate events. Instruction were written in image processing that if there is no eyelid movement establish for the definite phase of preset i.e. time greater than the time ofeye blinking of normal human then it considered as "blink" [10]. In this paper time is to be set as 5 seconds or more than it, as "blink event" is distinct from "normal eye blinking". The test is to be conducted for normal blinking of human eye.

\section{IR SENSOR}

To identify the drowsiness, eye blink sensor is used, which is IR based is shown in fig2. IR sensor consists of infrared transmitter and receiver. Infrared transmitter emits infrared rays. The transmitted IR rays are received by IR receiver [10].

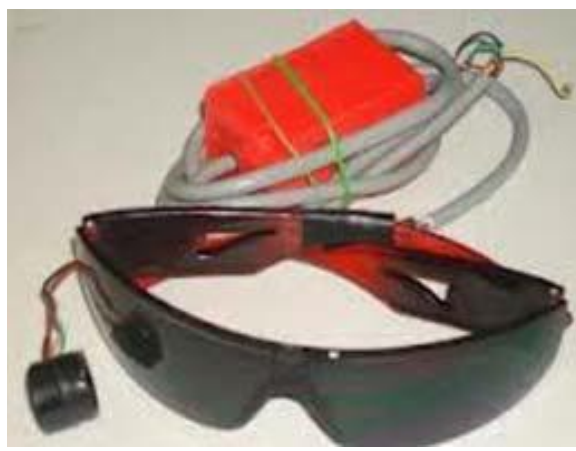

Fig 2: Eye blink sensor

The IR transmitter and IR receiver are arranged in parallel. When the signal is given, the IR sensor starts functioning and IR transmitter emits the infrared rays to the receiver. The comparator is coupled with IR receiver. The operational amplifier is attached to comparator.To the inverting input terminal of the comparator the reference voltage is given, the comparator is linked to receiver. When there is a disruption is present in the IR rays between sender (transmitter) and recipient (receiver), the IR receiver will not conduct. Hence the voltage at the inverting input terminal is lower than the voltage at the non inverting input. Therefore the output of comparator is high. The output voltage of comparator is given to microcontroller. When IR receiver receives the rays from transmitter, the IR receiver becomes conducting since the voltage at the non inverting terminal is lower than voltage at the inverting terminal. Therefore output of comparator is low. Hence the output of comparator is set to controller. This circuit is used for counting eyelid movement.

\section{ALCOHOL SENSOR}

The concentration of alcohol in our breathe is detected by using alcohol sensor. Sensor provides an analog output based on alcohol concentration. MQ-3 sensor is used as alcohol sensor is shown in fig 3. The alcohol sensor has an exceptional sensitivity and faster response time. The isobutane, propane, alcohol, cigarette, smoke, LNG are sensed by the sensor.

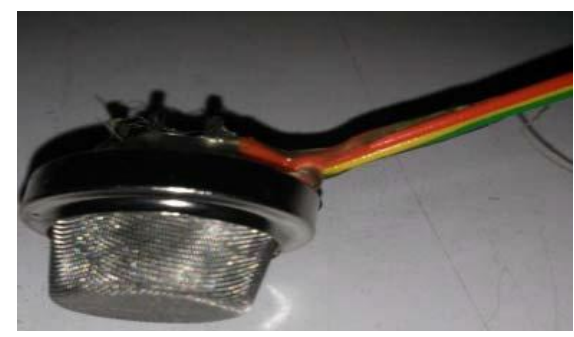

Fig 3: Alcohol sensor

The MQ-3 sensor has 6 pins. Here 4 pins are used to obtain signals and other two pins are used to providing heating current [9]. The MQ-3 sensor has a susceptible material of $\mathrm{SnO} 2$, which has a low conductivity in uncontaminated air [10]. If alcohol exists, the conductivity of sensor is higher along with the rising concentration of alcohol. 


\section{E. TILT Sensor}

According to variations in angular movement of an object, tilt sensor will produce an electrical signal. These sensors are used to enumerate tilt and slope within a narrow range of movement. Sometimes, the inclinometers are referred as tilt sensor because the sensor generates a signal but inclinometer generates both readout and a signal. It is used to detect the fall. It acts as a switch.
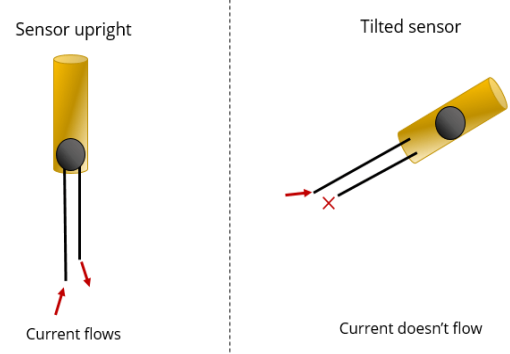

Fig 4: Tilt sensor

\section{F. LIGHT DEPENDENT RESISTOR}

A Light dependent resistor (LDR) is also called as a photo resistor or a cadmium sulfide $(\mathrm{CdS})$ cell is shown in fig 5. It is also known as a photoconductor.
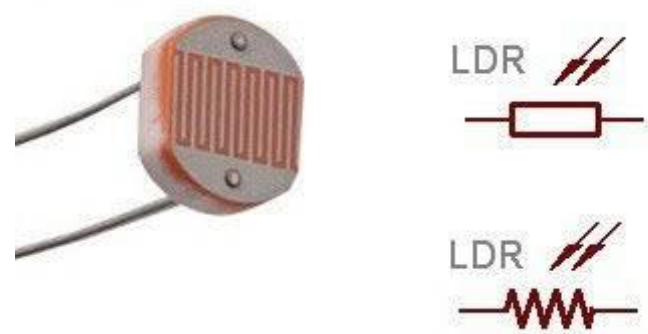

Fig 5: LDR

LDR is essentially a photocell that works on the principal of photoconductivity. It is a passive element is basically a resistor. LDR resistance increases with the increasing of illumination.
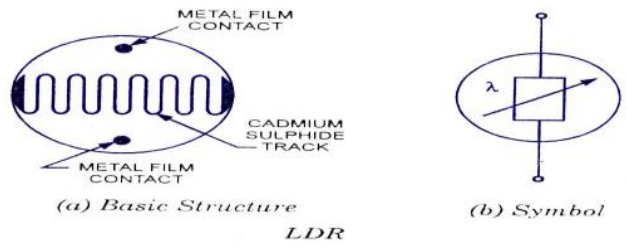

Fig 5.1:LDR working

The zigzag track shown in fig 5.1 is the cadmium sulphide $(\mathrm{CdS})$ film. The bottom as well as top metal films are attached to the terminal leads. It is designed such that to establish a maximum probable contact areas with the two metal films. The configuration is mounted in a plastic or resin case, to give access to outside light. The cadmium sulphide $(\mathrm{CdS})$ is the major component for the construction of LDR. It is used as the photoconductor and it consists of very few electrons. If the light is absent the LDR resistance is high. The electrons are liberated and the material conductivity is increases as illumination increases.If the illumination exceeds a specific frequency, the photons are captivated by the semiconductor and they give energy to electrons which is requisite to move to the conduction band. Due to this the free electrons or holes conduct electricity and thus reducing the resistance $(1<1$ Kilo ohm).

The following equation is used to show the relation between resistance as well as illumination are written as

$$
\mathrm{R}=\mathrm{A} \cdot \mathrm{E}^{\wedge} \mathrm{a}
$$

Here $\mathrm{E}$ is illumination (lux)

$\mathrm{R}$ is resistance (ohms)

$\mathrm{A}, \mathrm{a}$ are constants

In equation (1) ' $\mathrm{A}$ ' value depends on the $\mathrm{CdS}$ used along with on the manufacturing method. ' $A$ ' value range in between 0.7 and 9.9 .

\section{G. Buzzer Section}

To alert or indicate the completion of process, buzzer is used. Buzzer is for signify the start of the embedded system by alerting.

\section{H. LCD Section}

This LCD section is used to illustrate the status of the event. The Liquid Crystal Display (LCD) is used to display or prompt for necessary information.

\section{Global positioning system}

The location of any object or vehicle is determined by using satellite signals in Global positioning system (GPS) technology [5]. To find the receiver in 3D space three satellite signals are needed and for time accuracy the fourth satellite is used. The longitude, latitude and attitude parameters information are given by the GPS. By using longitude, latitude and attitude parameters location of any object can be estimated. In GPS technology, the communication is amid GPS transceiver and GPS satellite.

\section{J. Global system for mobile communication}

Global system for mobile communication (GSM) is the technology that underpins most of the world's mobile phone networks [5]. The GSM technology is extremely booming wireless technology along with a unique story of global attainment with increase in collaboration. GSM is the fastest mounting communication technology. GSM is digital cellular technology. For mobile voice and data service transmission and reception digital cellular technology is used. In GSM technology speed of data transfer is up to 9.6 kbps and allowing the broadcast of basic data services such as SMS.

\section{H. DC MOTOR}

A motor consists of a permanent magnetic field stator and a rotor. By using electromagnetic windings or permanent magnets, the magnetic field is maintained. For variable speed as well as for torque, DC motors are used.

\section{SOFTWARE TOOLS}

\section{A.Keil compiler}

To create software to be run on embedded system. It allows code to be written in assembly or in Cprogramming

\section{Published By:}

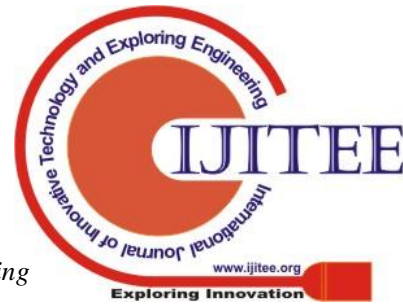


language and that code to be simulated on a computer before being loaded onto the controller unit. Micro vision 3 is an integrated Development Environment that helps to write a program. It compiles and runs embedded programs.

\section{B. Flash magic}

Flash magic is a PC tool for programming flash based microcontrollers from NXP using a serial or Ethernet protocol while in the target hardware. Streak magic is utilized to send the hex ode to the controller.

\section{DESCRIPTION}

For this work the requisite operating voltage for controller LPC2 148 is $3.3 \mathrm{~V}$. And power supply of 5V D.C. is required for the integrated chips. By using the Bridge Rectifier, the regulated $3.3 \mathrm{~V}$ is generated by stepping down the voltage from $230 \mathrm{~V}$ to $3.3 \mathrm{~V}$. Now the step downed A.C voltage is being rectified by using $1 \mathrm{~N} 4007$ diodes. The output voltage of bridge rectifier is filtered using a ' $\mathrm{C}$ ' filter. Now the rectified, filtered D.C. voltage is fed to the voltage regulator. To have a constant regulated voltage a voltage regulator is used. An electrolytic capacitor of 1000uf, the rectified, filtered and regulated voltage is again filtered for ripples. Now the output from this section is fed to microcontroller to supply operating voltage.

This project is basically aimed to detect drowsiness of driver. The intend of this project is to design an automated system for safety of driver from improper driving. The system consists of eye blink sensor, alcohol sensor, tilt sensor, LDR etc. The interfacing of devices to microcontroller is shown in fig6. The important block in this project is eye blink sensor which is IR based. IR rays are transmitted by transmitter into eye. The reflected IR rays from eye are received by receiver. If there is disruption in the IR rays amid transmitter and receiver means eye is in closed state, then the IR receiver becomes not conducting. Hence the voltage at the inverting input terminal of comparator is lower the non inverting input terminal voltage. Therefore comparator output is high and this voltage is fed to controller. It is used to determine the open or closing of eye of the driver. Hence whenever driver feels sleepy, eye blink sensor detect the closing position of eye and alert the driver by using buzzer and displayed in LCD. An eye blink is sensed by the eye blink sensor which is placed near to eye and this information is propagated in the form of pulses and it is transmitted to the ARM7 microcontroller. By using this information, the ARM7 controller will compare it with the normal eye blink which is written in code. If any atypical situation arises, the buzzer rings to alert driver. The alcohol sensor is used to detect whether the driver is drunken or not. If the driver is drunk, concentration of alcohol is detected by the MQ-3 sensor; the sensitive material of $\mathrm{SnO} 2$ conductivity is raised and provides an analog output to microcontroller. The microcontroller sends the warning signal through LCD which is shown in fig7.

The tilt sensor is used to detect that the vehicle is met with an accident or not. If vehicle is met with an accident, tilt sensor produce an electrical signal to microcontroller, by using GSM it gives information to the concern person that the vehicle is met with an accident. By using GPS the location of vehicle can be detected. The LDR is used to

detect the presence of light inside the vehicle. If light intensity is less in vehicle, the LDR resistance is high (mega ohms). As the light intensity increases on the LDR, electrons are liberated and conductivity is increases and lights will blow in vehicle.

The working of this system is explained in step by step process.

Step1: start process

Step2: If the data is sensed by eye blink sensor, process the sensed data by using comparator and operational amplifier

Step3: check the normal state or sleeping state of the driver

Step4: If it is normal state, output of comparator is GND and is given to LP2148 controller and buzzer is not ON

Step5: Else if sleeping mode is sensed, output of comparator is high is given microcontroller and buzzer is ON to alert the driver

Step6:If alcohol is also detected, conductivity of $\mathrm{SnO} 2$ raised and gives analog output to controller then it is displayed on LCD

Step7: If vehicle is met with an accident, the information is given through GSM to authorized person.

Step8: If the intensity of light is less, the lights will ON inside the vehicle.

\section{RESULT}

The figure 6 shows the interfacing of components which are used in this system. Here we have designed such that an eye blink is continuously monitored by the eye blink sensor.If eye is in closing position the buzzer is operated.

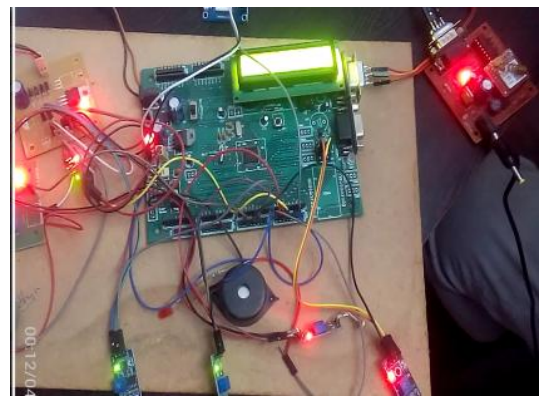

Fig 6: Driver drowsiness detection system

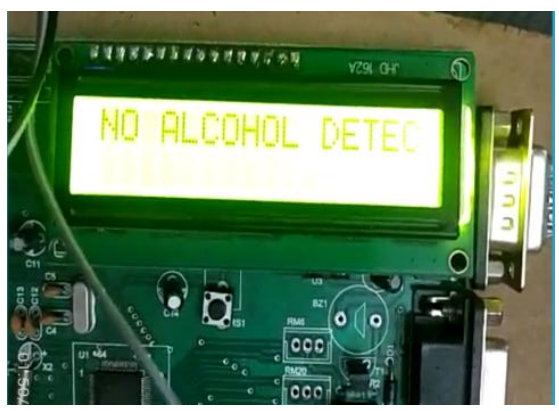

Fig7: Alcohol detection 
If alcohol is sensed by the alcohol sensor it warns the driver by displaying in LCD.

\section{ADVANTAGES}

The various advantages of the implemented system are mentioned below

1. Detection of drowsiness

2. Decreasing road accidents

3. No need of monitoring cameras or other devicesare attached or aimed at the driver.

4. This method is practically applicable.

\section{APPLICATIONS}

The drowsiness detection system can be used for different applications. One of them is heavy vehicles for example trucks, since the drivers of trucks have long driving periods. It can also be used for commercial vehicles. Many people use public transport facility for travelling. For their safety this system can be used in public vehicles. Heavy things are lifted by using cranes and transporting them to other places. So for overloaded cranes and mobile cranes this system can be used to avoid accidents related to drowsiness.

\section{CONCLUSION}

The driver drowsiness is analyzed and driver's drowsiness is detected and alert system is also designed. In this paper, the discussion regards the avoidance of accidents due to drowsiness is discussed with eye blink and corresponding system was developed. The project has been successfully tested.Advanced IC's and with the help of growing technologies like GSM and GPS the project has been successfully implemented.

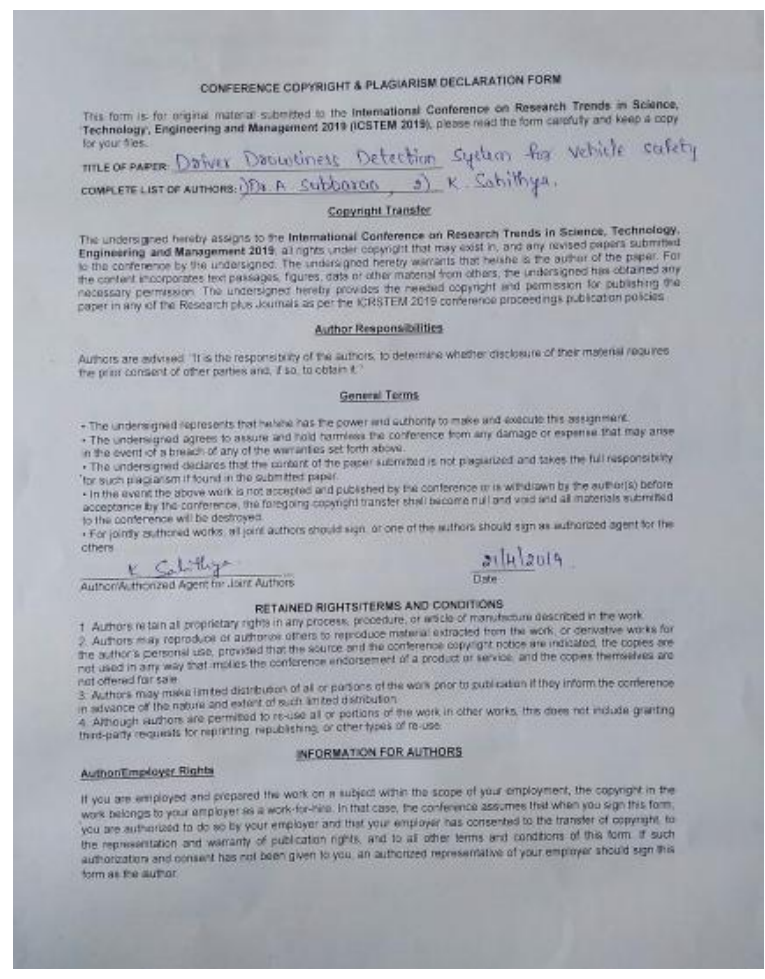

\section{ACKNOWLEDGMENT}

We are in deep gratitude to our department faculty who has always been supporting us through thick and thin as well as our project coordinator Mrs. CH. Sridevi, our H.O.D. Dr.J.Tarun Kumar, Associate Professor A.SubbaRao for their engulf support.

\section{REFERENCES}

1. M. Hemamalini, P. Muhilan"Accident preventionusing eye blink sensor”, vol 1, Issue L11, 2017.

2. RamalathaMarimuthu, A. Suresh, M. Alamelu and S.Kanagaraj "Driver fatigue detection using image processing and accident prevention", International journal of pure and applied mathematics, vol. 116, 2017.

3. TejaswiniJagdale, PradnyaJadhav, PrajaktaTotre,MayuraZadane, ShrilekhaMankhai "Driver drowsiness detection, alcohol detection and accident prevention", IJET, vol3 issue1, jan 2017

4. BappadityaMandal, LiyuanLiyuan Li, Gang Sam Wang and JieLin "Towards detection of bus driver fatigue based on robust visual analysis of eye state",IEEE transaction on intelligent transportation systems, 2016.

5. Suhaskatkar, Mahesh ManikKumbhar, PritiNavanathKadam"Accident prevention system using eye blink sensor", IRJET, Vol.03 Issue 05, 2016.

6. Tejasweenimusale, prof B,H. Pansambal, "Real time driver drowsiness detection system using image processing", IJREAM, Vol 02, Issue 08, 2016.

7. Omkar, RevatiBhor, PranjalMahajan, H.V. Kumbhar "Survey on Driver's drowsiness detection system", vol.132,2015.

8. Christy, Jasmeen Gill, "A Review: Driver drowsiness detection system", IJCST, Vol.3 Issue 4,jul-aug 2015.

9. Deepa K B, Chaitra M, Ankit Kumar Sharma, Sreedhar V S ,Prashanth Kumar H.K "Accident prevention by eye blinking sensor and alcohol detector", IJER, vol.no.4, issue no.7, 2015.

10. Rajasekar.R, Vivek Bharat Pattni, S.Vanangamudi "Drowsy driver sleeping device and driver alert system", IJSR, Vol.3 Issue 4,2014.

11. Swathi kale, RashmiBhadke,AnujaSali, NanasahebKadu'Drowsiness detection and warning system"IJARCST, Vol2, issue 2, 2014. 